\title{
Sir Ivor Batchelor
}

The knighthood conferred on Professor Ivor Batchelor in the Queen's Birthday Honours List will be acclaimed by his many friends and colleagues, both within psychiatry and outside it, as a fitting recognition.

Sir Ivor became the first holder of the Chair of Psychiatry in Dundee in 1962, and the high standing of psychiatry and psychiatric teaching in Dundee owes much indeed to his influence, vision, and wise counsel. But, of course, far beyond the local scene he has made many distinguished contributions, some beyond the boundaries of psychiatry itself. He was a member of the Porritt Committee, which reported on the review of the medical services in Great Britain; and through his past membership of the Scottish General Nursing Council and then the Briggs Committee he has helped to shape the future of education in the nursing profession. He has now been appointed a member of the new
Central Council for Nursing, Midwifery and Health Visiting. Sir Ivor's personal record of research earned him election as Fellow of the Royal Society of Edinburgh, and he has contributed to the work of the Medical Research Council as Chairman of the Council's Neurosciences Board. His chairmanship of the committee which reported on the staffing of mental handicap hospitals produced a constructive analysis which will surely have an enduring influence on British psychiatry for years to come. It was a further notable compliment to him when he was appointed a member of the Royal Commission on the National Health Service. And throughout these labours he has remained to his colleagues an inspiring clinician and teacher.

Peter Aungle JAMES MCHARG

\section{Election of Fellows}

The following Members have been elected to the Fellowship:

B. Alapin; D. Abrahamson; G. W. Ashcroft; B. R. Ballinger; E. D. Barlow; B. M. Barraclough; C. Berry; J. A. Birtchnell; A. A. Black; J. M. Black; M. R. Bond; J. A. Bonn; C. M. Brennan; I. G. Bronks; B. V. Burke; J. J. ByngHall; J. Caldbeck-Meenan; H. C. Cameron; J. A. Cameron; J. Candy; D. M. Carter; Elizabeth L. Cay; I. D. Chisholm; A. K. M. D. Chowdhury; J. Connolly; A. I. Cooklin; M. N. Cox; M. W. Coxon; T. J. Crow; R. L. Cundall; Joan Currah; C. Davies; Margaret E. B. Davies; M. H. Davies; D. H. Dick; J. H. Dowson; D. L. F. Dunleavy; Mrs Deepali Dutta; W. M. Easson; A. C. Fairburn; J. Farr; Eva A. Frommer; A. Galea; H. K. Gallagher; R. Gardner; M. J. Gay; A. I. M. Glen; C. Godber; A. S. R. Goonetilleke; L. K. Hemsi; Doris Hollander; F. A. S. Jensen; D. J. Jolley; M.
Joyston-Bechal; P. W. Kershaw; D. F. Klein; M. S. Lipsedge; G. A. Low-Beer; M. D. Lynch; R. MacDonald; A. S. MacVicar; R. V. Magnus; T. M. Maguire; A. A. Marinho; D. H. Marjoy; H. M. McBryde; J. L. McClure; J. McDonald; C. P. McEvedy; D. L. M. McNeill; P. I. Melia; D. W. Millard; D. H. Morgan; J. Muhangi; K. Myers; N. P. V. Nair; A. R. Nicol; F. D. Pascoe; Sheila M. W. Pittock; D. A. Player; I. G. Pryce; V. Ramachandran; A. V. N. Rao; Naomi S. Richman; H. K. Rose; B. M. Saran; B. G. Scally; J. C. Scott; D. Shaffer; J. G. Simeon; A. N. Singh; S. D. Soni; M. S. Spelman; W. B. Spry; W. J. Stanley; J. Steiner; G. Stores; M. Subramaniam; P. E. Sylvester; E. Szabadi; L. Tarlo; R. L. Tyson; E. L. Unwin; A. Verghese; J. D. Waliman; J. Warburton; J. L. S. Warnants; Marion C. Way; P. D. Wickenden; Marion E. F. Wollaston; E. G. Wooster; A. R. Worters; S. C. B. Yorke; M. E. York-Moore.

\section{Examination Results}

\section{Preliminiary Test-March 1981}

There were 325 candidates, of whom 150 passed.

Riadh Tawfiq Abed; Bassam Al-Ashhab; Chandranath Jayantha Amarasuriya; Dorothy Linda Andrews; Charles Amartey Aryiku; Don Susil Chandrasiri Attale; Awad Ibrahim Awad; Ibrahim Omar Awad;
Elizabeth Mary Bardsley; Susan Mary Barrow; Moustafa Abdel Basset; Anthony Walshaw Bateman; Sheela Biswas; Martin Hugh Briscoe; Simon Robert Britten; David Frank Brown; 